\title{
Angioedema Angioedema
}

\author{
Luisa Holguín-Gómez, ${ }^{1}$ Luz Adriana Vásquez-Ochoa, ${ }^{2}$ Ricardo Cardona ${ }^{3}$
}

\begin{abstract}
Angioedema is defined as edema of the skin or mucosa, including the respiratory and the gastrointestinal mucosa, which is self-limiting, and in most cases is completely resolved in less than 72 hours. It occurs due to increased permeability of the mucosal and submucosal capillaries and postcapillary venules, with resulting plasma extravasation. There are different types of angioedema: histaminergic (which may be mediated by immunoglobulin E), hereditary, from acquired C1 inhibitor deficiency, from angiotensin converting enzyme inhibitor, bradykinin-mediated, and nonhistaminergic idiopathic angioedema. Treatment depends on the cause of angioedema, age, and the frequency and severity of manifestations. The main measures are avoiding external triggers or causes, giving antihistamines, steroids, or adrenaline for histaminergic angioedema; replacing the deficient protein or blocking the action of bradykinin in C1 inhibitor deficiency and angioedema from angiotensin converting enzyme inhibitor.
\end{abstract}

Keywords: Angioedema; Histamine; Bradykinin; Idiopathic angioedema; Urticaria; Nonsteroidal anti-inflammatory drugs; Cyclooxygenase

Este artículo debe citarse como: Holguín-Gómez L, Vásquez-Ochoa LA, Cardona R. Angioedema. Rev Alerg Mex. 2016;63(4):373-384

${ }^{1}$ Universidad de Antioquia. Medellín, Antioquia, Colombia ${ }^{2}$ Hospital Pablo Tobón Uribe, Sección de Dermatología. Medellín, Antioquia, Colombia

${ }^{3}$ Universidad de Antioquia, Grupo de Alergología Clínica y Experimental, IPS Universitaria. Medellín, Antioquia, Colombia
Correspondencia: Luisa Holguín Gómez.

luisaholguing@gmail.com

Recibido: 2016-08-22

Aceptado: 2016-09-17 


\section{Resumen}

El angioedema se define como el edema de piel o mucosas, incluidas las de los tractos respiratorio y gastrointestinal, de carácter autolimitado, que en la mayoría de los casos se resuelve en forma completa en menos de 72 horas. Ocurre por aumento de la permeabilidad de los capilares mucosos, submucosos y vénulas poscapilares, con la consiguiente extravasación del plasma. Existen diferentes tipos de angioedema: el histaminérgico (que puede ser mediado o no por inmunoglobulina E), el hereditario, por déficit de $\mathrm{C} 1$ inhibidor adquirido, por inhibidores de la enzima convertidora de la angiotensina, mediados por bradiquininas y el angioedema no histaminérgico idiopático. El tratamiento depende de la causa del angioedema, la edad, frecuencia y gravedad de sus manifestaciones. Las principales medidas son evitar los desencadenantes o disparadores externos, la administración de antihistamínicos, esteroides o adrenalina en el angioedema histaminérgico; el reemplazo de la proteína deficiente o el bloqueo de la acción de la bradiquinina en el déficit de C1 inhibidor y en el angioedema por inhibidores de la enzima convertidora de la angiotensina.

Palabras clave: Angioedema; Histamina; Bradiquininas; Angioedema idiopático; Urticaria; Antiinflamatorios no esteroideos; Ciclooxigenasa

\author{
Abreviaturas y siglas \\ $\mathrm{AH}$, angioedema hereditario \\ AINE, antiinflamatorios no esteroideos \\ $\mathrm{AP}$, aminopeptidasa $\mathrm{P}$ \\ $\mathrm{C} 1-\mathrm{INH}$, inhibidor de $\mathrm{C} 1$ \\ DPP-IV, dipeptidil peptidasa IV \\ EPN, endopeptidasa neutra \\ EREA, enfermedad respiratoria exacerbada por AINE \\ $\mathrm{F} c \varepsilon R \mathrm{R}$, fracción $\mathrm{Fc}$ del receptor de alta afinidad para la lgE \\ FceRla, anticuerpos contra la subunidad alfa del receptor \\ de alta afinidad para la lgE
}

IECA, inhibidor de la enzima convertidora de la angiotensina $\lg E$, inmunoglobulina $E$

IgG, nmunoglobulina $\mathrm{G}$

MLCK, miosina de cadena ligera quinasa

PFC, plasma fresco congelado

PKC, proteína quinasa $\mathrm{C}$

SERPING1, serin protease inhibitor family member 1

$\mathrm{TSH}$, hormona estimulante de la tiroides

\section{Antecedentes}

El angioedema se define como el edema de piel o mucosas, incluidas las de los tractos respiratorio y gastrointestinal, de carácter autolimitado, con resolución completa en la mayoría de los casos, ${ }^{1,2}$ el cual ocurre por aumento de la permeabilidad de los capilares mucosos, submucosos y vénulas poscapilares, con la consiguiente extravasación del plasma. Los sitios más comúnmente afectados son cara, manos, pies y genitales. Su duración por lo general es menor de 72 horas. ${ }^{1,3}$

Existen diferentes tipos de angioedema: el histaminérgico, hereditario, por déficit de $\mathrm{C} 1$ inhibidor adquirido, por inhibidores de la enzima convertidora de la angiotensina (IECA) y el angioedema no histaminérgico idiopático. ${ }^{1}$ Sus causas dependen de la liberación de histamina o de bradiquininas.

Existen diferentes factores que intervienen en la liberación de histamina:

- El entrecruzamiento de la IgE específica dependiente de antígeno en la superficie de los mastocitos o basófilos.

- La activación autoinmune de las mismas células por una $\mathrm{IgG}$ anti-IgE.

- La activación de la IgG contra la fracción Fc del receptor de alta afinidad para la IgE (FceRI). 
- La mediación de complejos inmunes que causan activación del complemento y liberación de anafilotoxinas como $\mathrm{C} 3 \mathrm{a}, \mathrm{C} 4 \mathrm{a}$ y $\mathrm{C} 5 \mathrm{a} .{ }^{1,4}$

La bradiquinina es el mediador del angioedema asociado con los IECA, los cuales previenen la degradación de la bradiquinina.

Cuando existe deficiencia del $\mathrm{C} 1$ inhibidor ocurre una sobreproducción de bradiquininas y ausencia de inhibición de la calicreína y del factor XII activado. ${ }^{1}$

El tratamiento depende de la causa del angioedema y consiste en evitar los desencadenantes o disparadores externos, la administración de antihistamínicos, esteroides o adrenalina en el angioedema histaminérgico; reemplazo de la proteína deficiente o bloqueo de la acción de la bradiquinina en el déficit de $\mathrm{C} 1$ inhibidor y en el angioedema por IECA.

\section{Clasificación y enfoque diagnóstico}

El angioedema se clasifica según las manifestaciones cutáneas ${ }^{5}$ como:

\section{- Angioedema con habones}

Inducible: urticaria alérgica o urticaria física.

¿ Espontáneo: urticaria crónica.

- Angioedema sin habones

» Histaminérgico: alérgico o por liberación inespecífica de histamina (pseudoalérgico) y crónico recurrente.

» No histaminérgico: adquirido o hereditario.

También se puede clasificar según el mecanismo fisiopatológico que lo desarrolla ${ }^{1}$ (Cuadro 1).

Debe realizarse el diagnóstico diferencial con entidades diferentes al angioedema, como el edema asociado con cambios hormonales en las mujeres (facial, simétrico o en manos), el edema por insuficiencia venosa, falla cardiaca o enfermedad renal, el síndrome de vena cava superior y la queílitis granulomatosa. ${ }^{1,6}$

\section{Angioedema alérgico}

La exposición a un alérgeno produce una reacción de hipersensibilidad tipo $\mathrm{I}^{7}$ por la activación y proliferación de linfocitos Th2, los cuales migran a los nódulos linfáticos y estimulan el cambio de clase de la IgE en los linfocitos B. Dicha IgE se une a su receptor de alta afinidad en la membrana de los mastocitos y tras una nueva exposición al alérgeno se genera la activación de los mastocitos, con la posterior liberación de mediadores inflamatorios, responsables del angioedema y de otras manifestaciones clínicas. ${ }^{8}$ Los síntomas ocurren de minutos a 1 hora después de la exposición y los disparadores más comunes son alimentos, medicamentos o venenos de himenópteros. ${ }^{7}$ Por lo general, estas reacciones se relacionan con urticaria.

Los episodios intermitentes no ocurren con medicamentos que se toman diariamente y los síntomas pueden ser intermitentes o diarios dependiendo del alimento causal. Se sabe que los AINE generan la mayoría de las reacciones de hipersensibilidad en Latinoamérica y el mundo,,${ }^{9,10}$ sin embargo, la mediación $\operatorname{IgE}$ es poco frecuente y se ha demostrado que la inhibición de la COX-1 por los AINE junto con la presencia de un defecto regulatorio intrínseco (probablemente deficiencia de prostaglandina E2 y lipoxinas protectoras) desencadenan toda la cascada bioquímica que genera leucotrienos y la liberación de mediadores derivados de los eosinófilos y de los mastocitos. ${ }^{10,11}$

Existen métodos de tamizaje útiles, como las pruebas cutáneas y la medición de la IgE específica en sangre, sin embargo, la prueba de oro para el diagnóstico es la prueba de provocación doble ciego controlada con placebo. ${ }^{1}$

Cuadro 1. Clasificación etiológica del angioedema

1. Alérgico: por alimentos, medicamentos, venenos de himenópteros, etcétera.

2. Asociado con urticarias crónicas: espontáneo, inducible o vasculítico.

3. Asociado con enfermedades del tejido conectivo.

4. Asociado con sustancias con efecto liberador inespecífico de histamina: vancomicina, medios de contraste, etcétera.

5. Por inhibición de la COX-1: antiinflamatorios no esteroideos.

6. Mediado por bradiquininas: deficiencia de $\mathrm{C} 1-\mathrm{INH}$ (hereditaria o adquirida) y angioedema por IECA

7. Angioedema idiopático. 
Angioedema asociado con urticaria crónica espontánea 0 urticarias inducibles La urticaria crónica espontánea se define como la presencia de habones o angioedema diario o casi diario durante $\geq 6$ semanas, sin causa conocida. Afecta a $1 \%$ de la población general y es más frecuente en mujeres entre la tercera y quinta décadas de la vida. ${ }^{3,12}$ Aproximadamente $40 \%$ de los pacientes con urticaria crónica presenta angioedema y en $10 \%$ el angioedema es la manifestación principal.,

La fisiopatología de la urticaria crónica se desconoce, pero se cree que tiene un origen autoinmune ya que se han encontrado anticuerpos contra la subunidad alfa del receptor de alta afinidad para la $\operatorname{IgE}$ (FceRI $\alpha$ ) y contra la IgE propiamente con la activación de mastocitos y basófilos. A su vez, los complejos de IgG-FceRI $\alpha$ pueden activar el complemento por la vía clásica y generar $\mathrm{C} 5 \mathrm{a}$, una anafilotoxina cuyo receptor consiguiente se encuentra también en la membrana de los mastocitos, en los cuales genera más activación ${ }^{4}$ (Figura 1).

El diagnóstico de la urticaria crónica es clínico y solo se recomienda ordenar exámenes de marcadores de inflamación como hemoleucograma con velocidad de sedimentación globular o proteína $\mathrm{C}$ reactiva. El resto de los exámenes paraclínicos se solicitan según los hallazgos de la historia clínica (por ejemplo, TSH, Helicobacter pylori, biopsia, entre otros). ${ }^{3}$

Los hallazgos en el estudio histopatológico de la urticaria crónica se asemejan a los de una fase tardía de una inflamación alérgica, con infiltrado perivascular profundo y superficial, prominencia de linfocitos TCD4+, monocitos, neutrófilos y eosinófilos ${ }^{4} \mathrm{y}$ ausencia de linfocitos $\mathrm{B}$.

Las urticarias inducibles representan 15 a $25 \%$ de las urticarias crónicas y siempre requieren un disparador específico. ${ }^{13,14}$ En el grupo de las urticarias inducibles se incluyen las urticarias físicas: el dermografismo sintomático, la urticaria por frío o calor, la urticaria por presión tardía, la urticaria solar y el angioedema vibratorio; y las no físicas como las urticaria colinérgica, de contacto y la acuagénica. ${ }^{14}$ Frecuentemente los síntomas se limitan al área expuesta por el disparador específico y el diagnóstico se basa en la historia clínica y en los resultados de las pruebas de provocación determinadas. ${ }^{14}$

\section{Urticaria vasculítica}

La urticaria vasculítica puede interpretarse como una manifestación de una enfermedad del tejido conectivo o como una entidad aislada que afecta la piel. A diferencia de la urticaria/angioedema aguda o crónica produce una vasculitis leucocitoclástica con verdadera necrosis de pequeños vasos.

Predomina la urticaria sobre el angioedema y es característico que los habones duren de 24 a 48 horas y dejen una pigmentación residual. Con frecuencia son refractarios a los antihistamínicos. ${ }^{1}$

Ante una urticaria vasculítica con complemento bajo debe descartarse siempre lupus eritematoso sistémico (LES), aunque puede tratarse de síndrome urticarial vasculítico hipocomplementémico, descrito por McDuffie en 1973, caracterizado por lesiones urticariales de más de 24 horas de duración, asociado con compromiso renal, pulmonar, ocular, cardiaco y

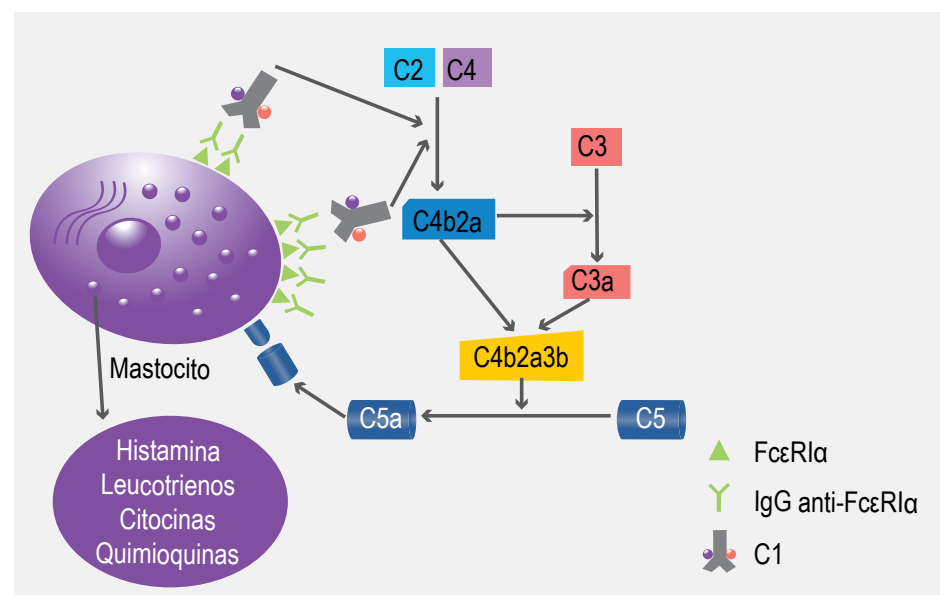

Figura 1. Fisiopatología de la urticaria crónica. Los anticuerpos IgG anti-FceRla se unen al receptor de alta afinidad de la lgE, formando complejos inmunes, con la posterior activación de los mastocitos; estos complejos son opsonizados por el complejo proteico $\mathrm{C} 1$ del complemento, con lo que se activa la vía clásica que finalmente genera C5a. El C5a se une a su receptor en la membrana de los mastocitos, con lo que provoca que estos se activen más. 
con alteraciones inmunológicas dadas por presencia de anticuerpos anti-C1q, con la consiguiente disminución de los niveles del C1q por activación de la vía clásica del complemento. Algunos autores lo consideran un tipo inusual de LES..$^{5-17}$

Otras entidades por considerar son las crioglobulinemias, poliarteritis nodosa, granulomatosis con poliangeítis y síndrome de Sjögren. ${ }^{1}$

\section{Inhibición de la ciclooxigenasa-1}

Existen dos formas de ciclooxigenasa (COX): COX-1, que se expresa de manera constitutiva en la mayoría de las células y tejidos; COX-2, inducida por citocinas y mediadores inflamatorios y cuya acción en mucho más lenta, aunque puede ser constitutiva en ciertas áreas como riñón y cerebro. ${ }^{18,19}$

El sitio activo COX está caracterizado por un canal estrecho y largo, hidrofóbico, sin embargo, el canal COX de la COX-2 es más amplio que el de la COX-1, debido a una diferencia en un único aminoácido en la posición 523, donde la isoleucina es sustituida por una valina. ${ }^{19}$

La mayoría de los AINE no selectivos compiten con el ácido araquidónico por la unión a la arginina en la posición 120 presente en ambos canales. Los inhibidores COX-2 selectivos interactúan con el canal y forman un apretado complejo, pero se unen al canal COX-1 de una manera reversible y con muy baja afinidad. ${ }^{19,20}$

La aspirina inhibe irreversiblemente a COX-1 y COX-2, por lo cual su duración de acción depende de la tasa de recambio de COX en los diferentes tejidos, siendo más larga y con efecto acumulativo en las plaquetas (de 8 a 12 días). Los AINE no aspirina inhiben competitivamente las COX en los sitios activados. ${ }^{19}$

La inhibición de la COX-1 por los antiinflamatorios no esteroideos - en conjunto con un defecto regulatorio intrínseco, probablemente deficiencia de prostaglandina E, la cual se encarga de la estabilización de las células inflamatorias (principalmente eosinófilos y mastocitos) y la 5-lipoxigenasa, por medio del receptor EP-2- dispara una cascada bioquímica que involucra la generación de leucotrienos, incremento de la expresión del receptor cysleucotrieno 1 y la liberación de mediadores inflamatorios derivados de eosinófilos y mastocitos. ${ }^{10,11}$

Los AINE se clasifican según su función farmacológica como inhibidores fuertes de la COX-1 (aspirina), inhibidores pobres de la COX-1 (acetaminofeno), inhibidores preferenciales de la COX2 (nimesulide) e inhibidores selectivos de COX-2 (celecoxib); la probabilidad y gravedad de la reacción depende del grado de inhibición de la COX-1, abarcando desde una urticaria con o sin angioedema hasta una anafilaxia. ${ }^{21}$

Existe de 10 a $30 \%$ de pacientes con urticaria crónica espontánea cuya enfermedad se exacerba con el consumo de AINE, en quienes se propone dicho mecanismo fisiopatológico. Así mismo, existe $10 \%$ de pacientes con la tríada de Samter (asma grave, rinosinusitis crónica con o sin poliposis nasal e intolerancia a AINE), actualmente catalogada como una enfermedad respiratoria exacerbada por AINE (EREA). ${ }^{11,22,23}$

Para el diagnóstico de hipersensibilidad a AINE es necesario realizar una historia clínica completa, que abarque aspectos relacionados con la descripción de los síntomas, medicamentos implicados, temporalidad, ruta de administración, episodios previos y presencia o no de enfermedades de base. ${ }^{22}$

En ocasiones, una historia clínica completa pudiera ser suficiente para establecer el diagnóstico, sin embargo, en numerosas ocasiones es necesaria la confirmación mediante pruebas de provocación orales, bronquiales o nasales bajo condiciones controladas; la prueba de provocación oral es el estándar de oro. ${ }^{24-28}$

Aunque todavía no está completamente estandarizado su uso, el parche con medicamentos pudiera ser útil en reacciones tardías, principalmente en dermatitis de contacto y eritema fijo por medicamentos; también se ha utilizado el fotoparche en pacientes con reacciones fotoalérgicas. ${ }^{23}$ Las provocaciones orales en caso de reacciones tardías solo están indicadas en exantema maculopapular, urticaria tardía y algunos casos de eritema fijo por medicamentos..$^{29,30}$

\section{Deficiencia de C1 inhibidor}

$\mathrm{El}$ angioedema hereditario (AH) resulta de la producción excesiva de bradiquinina, potente vasodilatador, generado por la calicreína tisular a partir del quininógeno de bajo peso molecular, como calidina y por la calicreína plasmática a partir del quininógeno de alto peso molecular durante la activación del sistema de contacto.

El inhibidor de $\mathrm{C} 1$ (C1-INH) es el más importante del sistema de contacto, también conocido 
como SERPING1 (serin protease inhibitor family member 1), inhibidor potente de la actividad de múltiples proteasas, entre ellas la calicreína.

La unión del C1-INH con su blanco produce un cambio conformacional en la molécula, modificando el sitio activo de este último y permitiendo la internalización del complejo a nivel intracelular para su posterior degradación (Figura 2)..$^{31,32}$

Existen tres tipos de AH (Cuadro 2):

- Tipo I: Corresponde a $85 \%$ de los casos, se caracteriza por bajos niveles de C1-INH, lo que genera bajos niveles funcionales.

- Tipo II: Representa $15 \%$ de los AH y se caracteriza por la presencia de una proteína disfuncional que se encuentra en cantidades normales o altas. Es identificado en pacientes afectados con $\mathrm{AH} .{ }^{33}$

Los AH tipo I y II son de transmisión autosómica dominante y están caracterizados por diferentes mutaciones en el gen SERPING1. De estos casos, 20 a $25 \%$ ocurre por una mutación de novo, con historia familiar negativa. ${ }^{33}$

- Tipo III: Son los AH con C1-INH normal, en los cuales se han postulado diferentes mecanismos fisiopatológicos, entre ellos mutaciones en el gen del factor XII de la coagulación y en los genes que codifican para enzimas que degradan las bradiquininas, como la aminopeptidasa $\mathrm{P}$ y la enzima convertidora de angiotensina (ECA). ${ }^{34-36}$

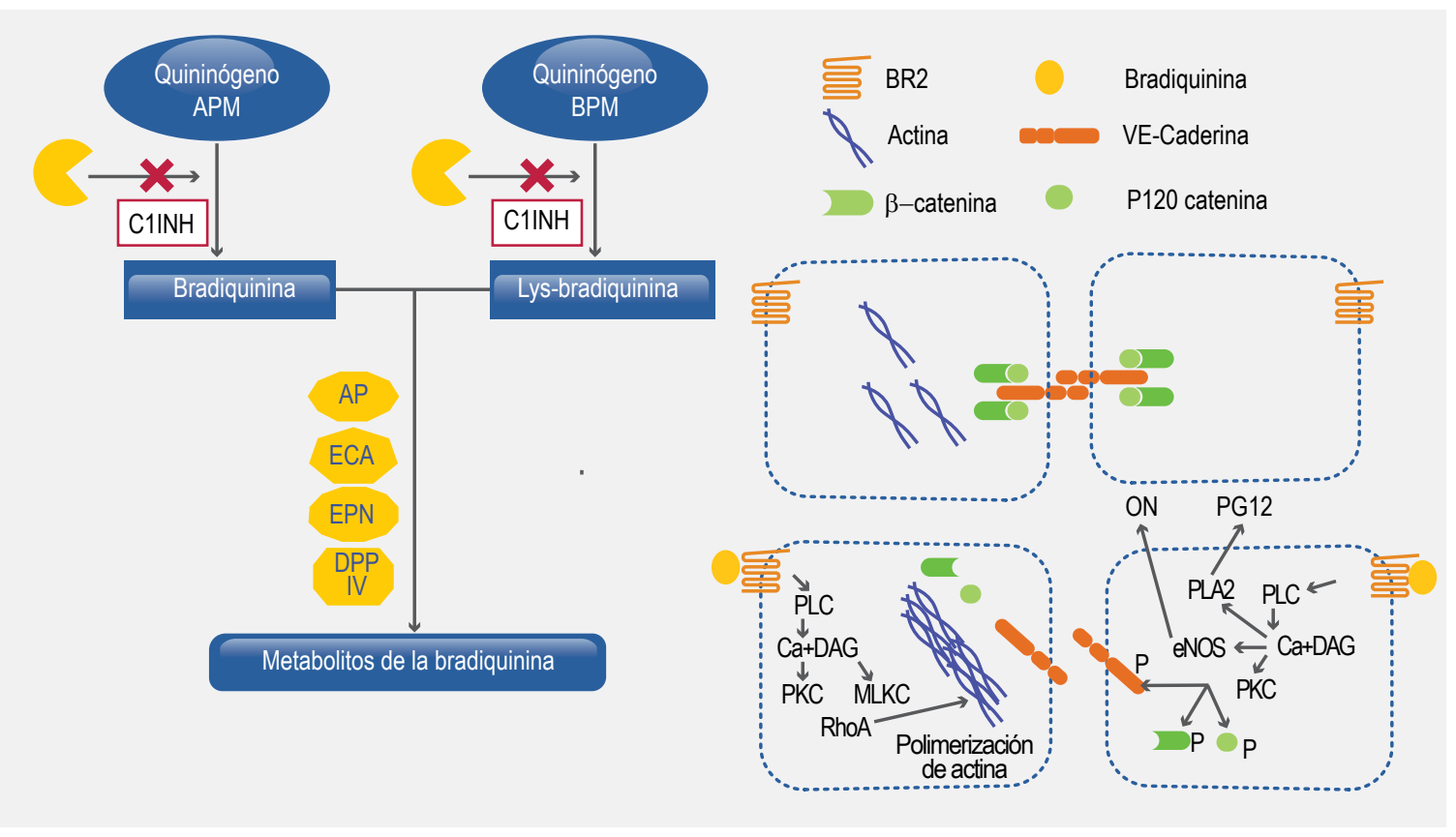

Figura 2. Formación y acción de la bradiquinina. La calicreína plasmática y la calicreína tisular generan bradiquinina y Lys-bradiquinina, respectivamente, que posteriormente son degradadas por las enzimas aminopeptidasa P (AP), enzima convertidora de angiotensina (ECA), endopeptidasa neutra (EPN) y dipeptidil peptidasa IV (DPP-IV). El C1-INH es el inhibidor más importante de la calicreína, modifica el sitio activo de la calicreína al producir un cambio conformacional en la estructura de la molécula y permite la internalización y degradación del complejo a nivel intracelular. La unión de la bradiquinina a su receptor en la membrana de las células endoteliales (BR2) activa una cascada de segundos mensajeros que aumenta el calcio intracelular y la formación de proteína quinasa C (PKC); esta última fosforila las proteínas VE-caderina, $\beta$-catenina y P120-catenina. A su vez, la activación de la GTPasa RhoA y la miosina de cadena ligera quinasa (MLCK) resulta en polimerización de la actina y retracción de las células endoteliales, aumentando los espacios intercelulares y el movimiento de líquido transcelular. 
En el AH, el angioedema es un edema asimétrico de piel y mucosas, sin prurito ni habones, que dura varios días (72 a 96 horas) y no mejora con antihistamínicos ni corticosteroides. ${ }^{37}$ Los principales desencadenantes son el trauma y el estrés emocional.

Generalmente los síntomas inician en la niñez, con edema de extremidades y abdomen, dolor abdominal severo, vómito y en ocasiones el depósito de líquido a un tercer espacio puede inducir hipotensión. Con frecuencia estos pacientes son sometidos a procedimientos invasivos innecesarios y la mortalidad puede llegar a $30 \%$ por compromiso laríngeo. ${ }^{38}$

El AH tipo III es más común en mujeres debido al papel que tienen los estrógenos, se manifiesta generalmente luego de la pubertad, con ataques menos frecuentes pero más severos, la distribución es más común en cara, lengua y menos en abdomen. ${ }^{39}$

El diagnóstico se confirma con niveles cuantitativos y funcionales del C1-INH inferiores a $50 \%$ del valor de referencia o mediante la medición de la actividad de la proteína. El diagnóstico debe basarse en dos mediciones separadas por 1 a 3 meses. El uso de los niveles del C4 más los niveles cuantitativos y funcionales del C1-INH para el diagnóstico del $\mathrm{AH}$ tiene una especificidad que oscila entre 98 y $100 \%$ y un valor predictivo negativo de $96 \%{ }^{40}$

\section{Angioedema por inhibidores de la enzima convertidora de angiotensina}

Se conoce que como parte del sistema calicreínaquinina, el quininógeno es convertido a bradiquinina mediante la calicreína y de esta forma la bradiquinina ejerce su acción en los receptores B1 y B2 localizados en el músculo liso y las células epiteliales vasculares. Así, el exceso en la producción de bradiquinina o de la actividad de sus receptores puede precipitar el aumento de la permeabilidad vascular, vasodilatación y acumulación de líquido intersticial. Cuando se inhibe la degradación de la bradiquinina ocurre el angioedema por IECA, ya que la enzima convertidora de la angiotensina cumple esta función junto con la aminopeptidasa P, la dipeptidil-peptidasa IV y la endopeptidasa neutra (Figura 3). ${ }^{12,41}$

No se puede predecir quién desarrollará angioedema al consumir IECA ni se ha establecido relación con la dosis, frecuencia o duración de la ingesta. Puede ocurrir hasta 6 meses después de la suspensión del medicamento. ${ }^{1,41}$

\section{Angioedema idiopático}

En forma característica, el angioedema idiopático se presenta episódicamente y ocurre pocas veces al año, es poco más frecuente en hombres y responde a antihistamínicos y esteroides. Es un diagnóstico de exclusión cuando no se identifican precipitantes exógenos, enfermedades del tejido conectivo ni anormalidades en el complemento. ${ }^{1}$

\section{Tratamiento}

El tratamiento del angioedema depende de su causa de base. En el angioedema alérgico consiste en evitar el alérgeno responsable y la administración de antihistamínicos, esteroides y adrenalina dependiendo de la severidad, evolución y localización, además de medidas de soporte (líquidos endovenosos, oxígeno, entre otras). ${ }^{3,42}$

$\mathrm{El}$ angioedema asociado con urticaria crónica o inducible debe ser tratado según las guías de tratamiento de la urticaria.

Los antihistamínicos son la piedra angular del tratamiento en las urticarias crónicas e inducibles; se prefieren los de segunda generación debido a los efectos anticolinérgicos y sedantes de los de primera generación, que pueden durar hasta 12 horas, mientras que el efecto antipruriginoso dura de 4 a 6 horas. ${ }^{3,43}$

El tratamiento debe iniciarse con una dosis usual diaria del antihistamínico y que se incrementa gradualmente hasta lograr el control de la enfermedad o alcanzar 4 veces la dosis convencional. ${ }^{44-46} \mathrm{Si}$ no se logra el control, la adición de 150 a $300 \mathrm{mg}$

\begin{tabular}{l|c|c|c|c|}
\hline \multicolumn{2}{l}{ Cuadro 2. Hallazgos de laboratorio en angioedema hereditario $(\mathrm{AH})$ por deficiencia del C1 inhibidor } \\
\hline \\
\hline AH tipo I & AH tipo II & AH tipo III & Adquirido \\
\hline C1-INH cuantitativo & Bajo & Normal o alto & Normal & Bajo \\
\hline C1-INH funcional & Bajo & Bajo & Normal & Bajo \\
\hline C4 & Bajo & Bajo & Normal & Bajo
\end{tabular}




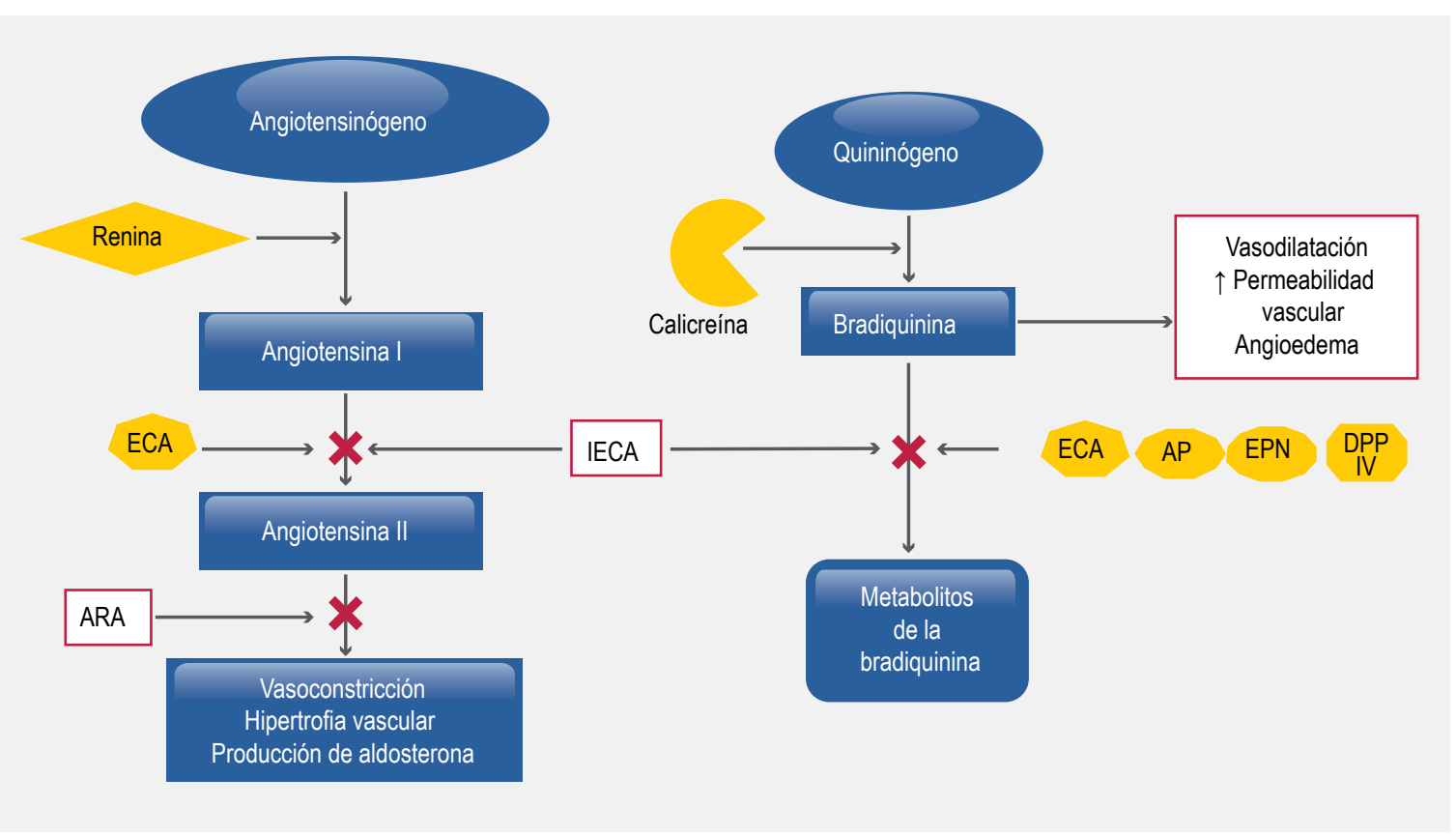

Figura 3. Relación del sistema de contacto con el sistema renina-angiotensina- aldosterona. Los IECA inhiben la degradación de la bradiquinina por la ECA y de esta manera la bradiquinina continúa ejerciendo su acción en los receptores B1 y B2 localizados en el músculo liso y las células epiteliales vasculares, causando vasodilatación, aumento de la permeabilidad vascular y angioedema.

de omalizumab cada mes ha demostrado ser efectiva. ${ }^{47-49}$ Existen otros medicamentos como la ciclosporina $\mathrm{A}$, la cual se ha asociado con alta incidencia de efectos adversos, y el montelukast, que ha venido en desuso por el bajo nivel de la evidencia disponible. En casos refractarios es necesario utilizar esteroides sistémicos. ${ }^{3,14}$

La urticaria vasculítica responde bien a la dapsona (100 mg al día) $)^{50}$ colchicina $(0.6 \mathrm{mg}$ dos o tres veces al día $)^{51} \mathrm{e}$ hidroxicloroquina (200 mg dos veces al día). ${ }^{52}$

En hipersensibilidad a AINE, el objetivo principal es proporcionar al paciente una opción analgésica, antipirética y antiinflamatoria segura; la confirmación debe realizarse por medio de una provocación oral bajo condiciones controladas. En pacientes con enfermedades cutáneas o respiratorias exacerbadas por AINE, el control de la enfermedad de base es fundamental. ${ }^{23}$

El angioedema mediado por bradiquininas no responde al manejo del angioedema histaminérgico. La principal medida de tratamiento del angioedema por IECA es la suspensión del medicamento desencadenante. ${ }^{41,53}$

En la deficiencia del C1-INH, el tratamiento profiláctico disponible en Colombia incluye el danazol como andrógeno atenuado con acción anabólica en el C1-INH, el cual ha demostrado ser seguro, incluso en niños, como profilaxis a corto plazo en dosis de 2.5 a $10 \mathrm{mg} / \mathrm{kg} /$ día (máximo $600 \mathrm{mg}$ al día, con inicio 5 días antes del procedimiento y con suspensión 2 a 5 días después del mismo). Es importante señalar que numerosos esquemas cortos se han asociado con efectos adversos importantes (virilización, labilidad emocional, alteraciones menstruales, acné, etcétera); no se recomiendan las dosis mayores a $200 \mathrm{mg}$ /día por periodos prolongados. El ácido tranexámico, derivado cíclico del ácido épsilon aminocaproico, ha sido empleado para profilaxis a corto plazo, con una dosis recomendada de $25 \mathrm{mg} / \mathrm{kg}$, dos o tres veces al día (de 3 a $6 \mathrm{~g}$ al día). ${ }^{33,40,54-56}$

Para el tratamiento agudo se dispone del plasma fresco congelado (PFC), que reemplaza a la proteína deficiente, sin embargo, existen reportes anecdóti- 
cos del empeoramiento debido a que se reemplazan también otras proteínas del complemento que se están consumiendo debido a la enfermedad, además del riesgo adicional inherente a la transfusión de hemoderivados; la dosis usual en adultos es de 2 a 4 unidades de PFC y en niños de $10 \mathrm{~mL} / \mathrm{kg}^{57}$

Por último, está el icatibant, un antagonista potente del receptor B2 de la bradiquinina, con afinidad y especificidad elevadas por los receptores de las quininas, disponible en jeringas precargadas de $30 \mathrm{mg}$ para administración subcutánea durante el ataque agudo, con una aplicación máxima de $90 \mathrm{mg} / 24$ horas. ${ }^{58-60}$
En otras partes del mundo existen otras opciones de tratamiento, algunas reemplazan la proteína deficiente, como el $\mathrm{C} 1$ inhibidor humano derivado del plasma y el $\mathrm{C} 1$ inhibidor recombinante; ${ }^{61-63}$ así como el ecallantide, un inhibidor reversible de la calicreína plasmática. ${ }^{64-66}$

La elección del tratamiento depende de la edad, frecuencia y gravedad de los ataques y la disponibilidad de los medicamentos. ${ }^{33,64}$

\section{Conflicto de intereses:}

Los autores declaran no tener conflicto de intereses.

\section{Referencias}

1. Kaplan AP. Angioedema. World Allergy Organ J. 2008;1(6):103-113. doi:10.1097/ WOX.0b013e31817aecbe

2. Mansi M, Zanichelli A, Coerezza A, Suffritti C, Wu MA, Vacchini R, et al. Presentation, diagnosis and treatment of angioedema without wheals: a retrospective analysis of a cohort of 1058 patients. J Intern Med. 2015 May;277(5):585-593. doi: 10.1111/joim.12304

3. Zuberbier T, Aberer W, Asero R, Bindslev-Jensen C, Brzoza Z, Canonica GW, et al. The EAACI/GA2LEN/ EDF/WAO Guideline for the definition, classification, diagnosis, and management of urticaria: The 2013 revision and update. Allergy Eur J Allergy Clin Immunol. 2014;69(7):868-887. doi:10.1111/all.12313

4. Kaplan AP, Greaves M. Pathogenesis of chronic urticaria. Clin Exp Allergy. 2009;39(6):777-787. doi:10.1111/j.1365-2222.2009.03256.x.

5. Wu MA, Perego F, Zanichelli A, Cicardi M. Angioedema phenotypes: Disease expression and classification. Clin Rev Allergy Immunol. 2016;51(2):1-8. doi:10.1007/s12016-016-8541-z

6. Mahendran K, Padmini G, Murugesan R, Srikumar A. Acute allergic angioedema of upper lip. J Conserv Dent. 2016;19(3):285. doi:10.4103/0972-0707.181949

7. Temiño VM, Peebles RS. The spectrum and treatment of angioedema. Am J Med. 2008;121(4):282-286. doi:10.1016/j.amjmed.2007.09.024

8. Abbas AK, Lichtman AH, Pillai S. Cellular and molecular immunology. Atlanta, GA, USA: Elsevier; 2014.

9. Jares EJ, Sánchez-Borges M, Cardona-Villa R, Ensina LF, Arias-Cruz A, Gómez M, et al. Multinational experience with hypersensitivity drug reactions in Latin America. Ann Allergy, Asthma Immunol. 2014;113(3):282-289. doi:10.1016/j.anai.2014.06.019

10. Torres MJ, Barrionuevo E, Kowalski M, Blanca M. Hypersensitivity reactions to non-steroidal antiinflammatory drugs. Immunol Allergy Clin North Am. 2014;34:507-524. doi:10.1016/j.iac.2014.04.001

11. Cornejo-García JA, Blanca-López N, Dona I, Andreu I, Agúndez JA, Carballo M, et al. Hypersensitivity reactions to non-steroidal anti-inflammatory drugs. Curr Drug Metab. 2009;10(9):971-980. doi:10.1016/j. iac.2014.04.001

12. Adkinson NF, Bochner BS, Burks W, Busse, William W, Holgate ST, et al. Middleton's allergy principles and practice. Octava edición. Atlanta, GA, USA: Elsevier; 2014.

13. Abajian M, Mlynek A, Maurer M. Physical urticaria. Curr Allergy Asthma Rep. 2012;12(4):281-287. doi:10.1007/s11882-012-0269-0

14. Magerl M, Altrichter S, Borzova E, Giménez-Arnau A, Grattan CEH, Lawlor F, et al. The definition, diagnostic testing and management of chronic inducible urticarias. Update and revision of the EAACI/ GA2LEN/EDF/UNEV 201 consensus recommendations 2016 update and revision. Allergy. 2016;71:780702. doi:10.1111/all.12884 
15. Parra V, Darío-Aguirre H, Daza RA, Mora SA, Peñaranda E. Lupus eritematoso sistémico versus urticaria vasculítica hipocomplementémica: un dilema diagnóstico en la práctica clínica. Rev Colomb Reumatol. 2015;22(3):168-173.

16. Chang S, CarrW. Urticarial vasculitis. Allergy Asthma Proc. 2007;28(1):97-100. doi:10.2500/aap.2007.28.2972

17. Pulido-Pérez A, Avilés-Izquierdo JA, Suárez-Fernández R. Vasculitis cutáneas. Actas Dermosifiliogr. 2012;103(3):179-191. doi:10.1016/j.ad.2011.06.00.

18. Maitra A. Endocrine system. En: Kumar V, Abbas AK, Aster JC, editores. Robbins basic pathology. Novena edición. Philadelphia: Elsevier Saunders; 2013. p. 715-764.

19. Tacconelli S, Capone ML, Patrignani P. Clinical pharmacology of novel selective COX-2 inhibitors. Curr Pharm Des. 2004;10(6):589-601. doi:10.2174/1381612043453108

20. Cryer B, Feldman M. Cyclooxygenase-1 and cyclooxygenase-2 selectivity of widely used nonsteroidal anti-inflammatory drugs. Am J Med. 1998;104(5):413-421. doi:10.1016/S0002-9343(98)00091-6

21. Lee RU, Stevenson DD. Aspirin-exacerbated respiratory disease: evaluation and management. Allergy Asthma Immunol Res. 2011;3(1):3. doi:10.4168/aair.2011.3.1.3.

22. Kowalski ML, Woessner K, Sanak M. Approaches to the diagnosis and management of patients with a history of nonsteroidal anti-inflammatory drug-related urticaria and angioedema. J Allergy Clin Immunol. 2015;136(2):245-251. doi:10.1016/j.jaci.2015.06.021.

23. Kowalski ML, Asero R, Bavbek S, Blanca M, Blanca-López N, Bochenek G, et al. Classification and practical approach to the diagnosis and management of hypersensitivity to nonsteroidal anti-inflammatory drugs. Allergy Eur J Allergy Clin Immunol. 2013;68(10):1219-1232. doi:10.1111/all.12260.

24. Milewski M, Mastalerz L, Nizankowska E, Szczeklik A. Nasal provocation test with lysine-aspirin for diagnosis of aspirin-sensitive asthma. J Allergy Clin Immunol. 1998;101(5):581-586. doi:10.1016/S00916749(98)70163-0

25. Nizankowska E, Bestynska-Krypel A, Cmiel A, Szczeklik A. Oral and bronchial provocation tests with aspirin for diagnosis of aspirin-induced asthma. Eur Respir J. 2000;15(5):863-869.

26. Nizankowska-Mogilnicka E, Bochenek G, Mastalerz L, Swierczyńska M, Picado C, Scadding G, et al. EAACI/GA2LEN guideline: aspirin provocation tests for diagnosis of aspirin hypersensitivity. Allergy. 2007;62(10):1111-1118. doi:10.1111/j.1398-9995.2007.01409.x

27. Macy E, Bernstein JA, Castells MC, Gawchik SM, Lee TH, Settipane RA, et al. Aspirin challenge and desensitization for aspirin-exacerbated respiratory disease: a practice paper. Ann Allergy Asthma Immunol. 2007;98(2):172-174. doi:10.1016/S1081-1206(10)60692-8

28. Asero R. Oral aspirin challenges in patients with a history of intolerance to single non-steroidal antiinflammatory drugs. Clin Exp Allergy. 2005;35(6):713-716. doi:10.1111/j.1365-2222.2005.2228.x

29. Barbaud A. Drug patch testing in systemic cutaneous drug allergy. Toxicology. 2005;209(2):209-216. doi:10.1016/j.tox.2004.12.024.

30. Barbaud A, Goncalo M, Bruynzeel D, Bircher A. Guidelines for performing skin tests with drugs in the investigation of cutaneous adverse drug reactions. Contact Dermatitis. 2001;45(6):321-328.

31. Cugno M, Zanichelli A, Foieni F, Caccia S, Cicardi M. C1-inhibitor deficiency and angioedema: molecular mechanisms and clinical progress. Trends Mol Med. 2009;15(2):69-78. doi:10.1016/j.molmed.2008.12.001

32. Zeerleder S, Levi M. Hereditary and acquired C1-inhibitor-dependent angioedema: from pathophysiology to treatment. Ann Med. 2016;3890:1-12. doi:10.3109/07853890.2016.1162909

33. Craig TJ, Pürsün EA, Bork K, Bowen T, Boysen H, Farkas H, et al. WAO Guideline for the management of hereditary angioedema. World Allergy Organ J. 2012;5(12):182-199. doi:10.1097/ WOX.0b013e318279affa

34. Riedl MA. Hereditary angioedema with normal C1-INH (HAE Type III). J Allergy Clin Immunol Pract. 2013;1(5):427-432. doi:10.1016/j.jaip.2013.06.004.

35. Drouet C, Désormeaux A, Robillard J, Ponard D, Bouillet L, Martin L, et al. Metallopeptidase activities in hereditary angioedema: Effect of androgen prophylaxis on plasma aminopeptidase P. J Allergy Clin Immunol. 2008;121(2):429-433. doi:10.1016/j.jaci.2007.10.048 
36. Davis AE 3rd. The pathophysiology of hereditary angioedema. Clin Immunol. 2005;114(1):3-9.

37. Walford HH, Zuraw BL. Current update on cellular and molecular mechanisms of hereditary angioedema. Ann Allergy Asthma Immunol. 2014;112(5):413-418. doi:10.1016/j.anai.2013.12.02

38. Atkinson JP, Cicardi M, Zuraw BL, Saini S FA. Hereditary angioedema: Epidemiology, clinical manifestations, exacerbating factors, and prognosis. UpToDate. 2014:1-11.

39. Bork K, Wulff K, Hardt J, Witzke G, Staubach P. Hereditary angioedema caused by missense mutations in the factor XII gene: Clinical features, trigger factors, and therapy. J Allergy Clin Immunol. 2009;124(1):129-134. doi:10.1016/j.jaci.2009.03.038

40. Cicardi M, Aberer W, Banerji A, Bas M, Bernstein JA, Bork K, et al. Classification, diagnosis, and approach to treatment for angioedema: Consensus report from the Hereditary Angioedema International Working Group. Allergy. 2014;69(5):602-616. doi:10.1111/all.12380

41. Scalese MJ, Reinaker TS. Pharmacologic management of angioedema induced by angiotensinconverting enzyme inhibitors. Am J Heal Pharm. 2016;73(12):873-879. doi:10.2146/ajhp150482

42. Wilkerson RG. Angioedema in the emergency department: an evidence-based review. Emerg Med Pract. 2012;14(11):1-21.

43. Church MK, Maurer M, Simons FER, Bindslev-Jensen C, van Cauwenberge P, Bousquet J, et al. Risk of first-generation $\mathrm{H}(1)$-antihistamines: A GA(2)LEN position paper. Allergy Eur J Allergy Clin Immunol. 2010;65(4):459-466. doi:10.1111/j.1398-9995.2009.02325.x

44. Staevska M, Popov TA, Kralimarkova T, Lazarova C, Kraeva S, Popova D, et al. The effectiveness of levocetirizine and desloratadine in up to 4 times conventional doses in difficult-to-treat urticaria. J Allergy Clin Immunol. 2010;125(3):676-682. doi:10.1016/j.jaci.2009.11.047

45. Krause K, Spohr A, Zuberbier T, Church MK, Maurer M. Up-dosing with bilastine results in improved effectiveness in cold contact urticaria. Allergy Eur J Allergy Clin Immunol. 2013;68(7):921-928.

46. Tubert-Brohman I, Sherman W, Repasky M, Beuming T. Improved docking of polypeptides with glide. J Chem Inf Model. 2013;53(7):1689-1699. doi: 10.1021/ci400128m

47. Spector SL, Tan RA. Effect of omalizumab on patients with chronic urticaria. Ann Allergy Asthma Immunol. 2007;99(2):190-193. doi:10.1016/S1081-1206(10)60644-8

48. Büyüköztürk $S$, Gelincik A, Demirtürk M, Kocaturk $E$, Çolakoğlu B, Dal M. Omalizumab markedly improves urticaria activity scores and quality of life scores in chronic spontaneous urticaria patients: A real life survey. J Dermatol. 2012;39(5):439-442. doi:10.1111/j.1346-8138.2011.01473.x

49. Metz M, Altrichter S, Ardelean E, Kessler B, Krause K, Magerl M, et al. Anti-immunoglobulin E treatment of patients with recalcitrant physical urticaria. Int Arch Allergy Immunol. 2011;154(2):177-180.

50. Nurnberg W, Grabbe J, Czarnetzki BM. Urticarial vasculitis syndrome effectively treated with dapsone and pentoxifylline. Acta Derm Venereol. 1995;75(1):54-56.

51. Wiles JC, Hansen RC, Lynch PJ. Urticarial vasculitis treated with colchicine. Arch Dermatol. 1985;121(6):802-805. doi:10.1001/archderm.1985.01660060116035.

52. Lopez LI, Davis KC, Kohler PF, Schocket AL. The hypocomplementemic urticarial-vasculitis syndrome: therapeutic response to hydroxychloroquine. J Allergy Clin Immunol. 1984;73(5 Pt 1):600-603.

53. Caballero T, Baeza ML, Cabañas R, Campos A, Cimbollek S, Gómez-Traseira C, et al. Consensus statement on the diagnosis, management, and treatment of angioedema mediated by bradykinin. Part I. Classification, epidemiology, pathophysiology, genetics, clinical symptoms, and diagnosis. J Investig Allergol Clin Immunol. 2011;21(5):333-347.

54. Bowen T, Cicardi M, Bork K, Zuraw B, Frank M, Ritchie B, et al. Hereditary angiodema: A current state-of-the-art review, VII: Canadian Hungarian 2007 International Consensus Algorithm for the Diagnosis, Therapy, and Management of Hereditary Angioedema. Ann Allergy, Asthma Immunol. 2008;100(1):S30-S40. doi:10.1016/S1081-1206(10)60584-4

55. Bowen T, Cicardi M, Farkas H, Bork K, Kreuz W, Zingale L, et al. Canadian 2003 International Consensus Algorithm for the Diagnosis, Therapy, and Management of Hereditary Angioedema. J Allergy Clin Immunol. 2004;114(3):629-637. doi:10.1016/j.jaci.2004.06.043. 
56. Gompels MM, Lock RJ, Abinun M, Bethune CA, Davies G, Grattan C, et al. C1 inhibitor deficiency: Consensus document. Clin Exp Immunol. 2005;139(3):379-394. doi:10.1111/j.1365-2249.2005.02726.x

57. Charignon D, Ghannam A, Defendi F, Ponard D, Monnier N, López Trascasa M, et al. Hereditary angioedema with F12 mutation: Factors modifying the clinical phenotype. Allergy. 2014;69(12):16591665. doi: 10.1111/all.12515

58. Charignon D, Späth P, Martin L, Drouet C. Icatibant, the bradykinin B2 receptor antagonist with target to the interconnected kinin systems. Expert Opin Pharmacother. 2012;13(15):2233-2247. doi:10.1517/146 56566.2012.723692

59. Cicardi M, Banerji A, Bracho F, Malbrán A, Rosenkranz B, Riedl M, et al. Icatibant, a new bradykininreceptor antagonist, in hereditary angioedema. N Engl J Med. 2010;363(6):532-541. doi:10.1056/ NEJMoa0906393

60. Lumry WR, Li HH, Levy RJ, Potter PC, Farkas H, Moldovan D, et al. Randomized placebo-controlled trial of the bradykinin B2 receptor antagonist icatibant for the treatment of acute attacks of hereditary angioedema: The FAST-3 trial. Ann Allergy Asthma Immunol. 2011;107(6):529-537. doi:10.1016/j. anai.2011.08.015

61. Czaller I, Visy B, Csuka D, Füst G, Tóth F, Farkas H. The natural history of hereditary angioedema and the impact of treatment with human $\mathrm{C} 1$-inhibitor concentrate during pregnancy: A long-term survey. Eur J Obstet Gynecol Reprod Biol. 2010;152(1):44-49. doi:10.1016/j.ejogrb.2010.05.008

62. Craig TJ, Levy RJ, Wasserman RL, Bewtra AK, Hurewitz D, Obtułowicz K, et al. Efficacy of human C1 esterase inhibitor concentrate compared with placebo in acute hereditary angioedema attacks. J Allergy Clin Immunol. 2009;124(4):801-808. doi:10.1016/j.jaci.2009.07.017

63. Craig TJ, Bewtra AK, Bahna SL, Hurewitz D, Schneider LC, Levy RJ, et al. C1 esterase inhibitor concentrate in 1085 hereditary angioedema attacks-final results of the I.M.P.A.C.T.2 study. Allergy Eur J Allergy Clin Immunol. 2011;66(12):1604-1611. doi:10.1111/j.1398-9995.2011.02702.x

64. Xu YY, Buyantseva LV, Agarwal NS, Olivieri K, Zhi YX, Craig TJ. Update on treatment of hereditary angioedema. Clin Exp Allergy. 2013;43(4):395-405. doi:10.1111/cea.12080

65. Riedl M, Campion M, Horn PT, Pullman WE. Response time for ecallantide treatment of acute hereditary angioedema attacks. Ann Allergy, Asthma Immunol. 2010;105(6):430-436.e2. doi:10.1016/j. anai.2010.09.005

66. Sheffer AL, Campion M, Levy RJ, Li HH, Horn PT, Pullman WE. Ecallantide (DX-88) for acute hereditary angioedema attacks: Integrated analysis of 2 double-blind, phase 3 studies. J Allergy Clin Immunol. 2011;128(1):153-159. doi:10.1016/j.jaci.2011.03.006 